\title{
Pulmonary Hypertension, CTCAE
}

National Cancer Institute

\section{Source}

National Cancer Institute. Pulmonary Hypertension, CT CAE. NCI Thesaurus. Code C55068.

A disorder characterized by an increase in pressure within the pulmonary circulation due to lung or heart disorder. 